Function was investigated by analysis of suppressive activity of Treg on proliferation of autologous effector T cells in vitro utilizing suppression assays.

Results: First results at the time-point of switching to anti-IL-17 treatment demonstrated PsA to be an IL-17-driven T cell-mediated autoimmune disorder, as proportions of T cells with Th17 phenotype were increased in PsA compared to controls (CCR6+lL-17+4.9\% vs. $0.8 \%$ of $\mathrm{CD} 4+$ ) and FoxP3+ Treg cells (CD25brightFoxP $3+0.2 \%$ vs. $0.4 \%$ of CD4+) were decreased. Higher proportions of FoxP3+ T cells expressing the Th17-characteristic chemokine receptor CCR6 were found in PsA (4.8\% vs. $2.7 \%$ of CD4+), as well as higher proportions of pro-apoptotic CD95-expressing FoxP3+T cells ( $9.8 \%$ vs. $2.8 \%$ of CD4+). Less suppression of autologous effector $\mathrm{T}$ cells co-cultured with CD25+ Treg cells was found in PsA compared to controls $(22.2 \%$ vs. $28.3 \%$ reduction of proliferative activity), whereas CD25- helper $\mathrm{T}$ cells did not contribute to the suppression of effectors in PsA and only minimally in controls. Intracellular IL-10 production in Tregs, a key cytokine of Treg-associated regulation of inflammation, was similar between PsA and controls, although a trend to lower CTLA-4 expression involved in inhibition of co-stimulation was found in PsA.

Conclusion: The current results indicate a skewed $T$ cell balance towards Th17 cells and Treg cells showing Th17-like features in samples of PsA unsuccessfully pre-treated with different biologics recommending them for a switch to a therapy with selective inhibition of IL-17. Longitudinal results regarding the reconstitution and maintenance of Treg function in those PsA patients have to be awaited.

Disclosure of Interests: Timotheos Christoforou: None declared, Giovanni Almanzar Grant/research support from: Pfizer, Franziska Brauneiser: None declared, Nils Buschmann: None declared, Martin Feuchtenberger Consultant of: Abbvie, BMS, Chugai, Sanofi, Speakers bureau: Abbvie, BMS, Celgene, Chugai, Jansen-Cilag, Lilly, Pfizer, Roche, Sanofi, UCB, Marc Schmalzing Consultant of: Paid consultant for Hexal AG, Hans-Peter Tony Consultant of: AbbVie, Astra-Zeneca, BMS, Chugai, Janssen, Lilly, MSD, Novartis, Pfizer, Roche, Sanofi, Matthias Goebeler: None declared, Martina Prelog Grant/research support from: Chugai, Sobi, Novartis, Pfizer, Baxter, Consultant of: GSK, Pfizer, Novartis, MSD, Baxter, Sobi, Johnson, Speakers bureau: GSK, Pfizer, Novartis, MSD, Baxter, Sobi, Johnson DOI: 10.1136/annrheumdis-2020-eular.6389

\section{AB0041 CD8+ T CELLS HAVE AN ELEVATED PROLIFERATIVE CAPACITY IN GIANT CELL ARTERITIS}

R. Reitsema ${ }^{1}$, R. Hid Cadena ${ }^{2}$, W. Abdulahad ${ }^{1,2}$, A. Boots ${ }^{1}$, P. Heeringa ${ }^{2}$, E. Brouwer ${ }^{1}{ }^{1}$ University of Groningen, University Medical Center Groningen, Rheumatology and Clinical Immunology, Groningen, Netherlands; ${ }^{2}$ University of Groningen, University Medical Center Groningen, Pathology and Medical Biology, Groningen, Netherlands

Background: Giant cell arteritis (GCA) is the most frequent form of systemic vasculitis affecting the large- and medium-sized vessels. The involvement of innate immune cells and CD4+ T cells in the pathogenesis of GCA has been extensively studied. Interestingly, recent findings suggest a role for CD8+ T cells in disease development (1). However, CD8+ subsets and their functional capacities have not yet been studied in detail.

Objectives: This study aims to characterize the phenotype and proliferative capacity of CD8+ T cells in newly diagnosed GCA patients and GCA patients in remission compared to healthy age- and sex- matched controls.

Methods: To determine the phenotype of CD8+ T cells in GCA, newly diagnosed, untreated GCA patients (baseline, $n=14$ ), GCA patients in stable glucocorticoid-free remission (GC-FR, $n=10$ ) and age- and sex-matched healthy controls (HCs, $\mathrm{n}=18$ ) were enrolled. Peripheral blood mononuclear cells (PBMCs) were stained with fluorochrome-conjugated antibodies directed against CD3, CD4, CD8, CCR7, CD45RO, Ki-67, CD69 and CD25 and analyzed by flow cytometry. The following differentiation subsets were defined: CD8+ $T$ naive (CD45RO-CCR7+), central memory ( $\left.T_{C M}, C D 45 R O+C C R 7+\right)$, effector memory ( $\left.T_{E M}, C D 45 R O+C C R 7-\right)$ and effector memory re-expressing CD45RA ( $T_{E M R A}$, CD45RO-CCR7-) cells. Secondly, the proliferative capacity of CD8+ T cells was determined in isolated CD3+ T cells of 10 GCA baseline, 10 GCA GC-FR patients and $19 \mathrm{HCs}$ after 5 days of stimulation with plate-bound anti-CD3 or anti-CD3 plus soluble anti-CD28 using a dye-based proliferation assay.

Results: A reduced frequency of $C D 8+T_{E M}$ cells was found in GCA baseline patients compared to $\mathrm{HCs}(\mathrm{p}=0.025)$. Furthermore, a higher frequency of Ki-67+ cells was detected among CD8 $+\mathrm{T}_{\mathrm{EM}}$ cells in GCA baseline patients than in $\mathrm{HCs}$ $(\mathrm{p}=0.0007)$, suggesting a higher proliferative activity in vivo. In addition, in vitro stimulation with anti-CD3 and anti-CD3+anti-CD28 led to higher percentages of divided CD8+ T cells in GCA baseline and GC-FR patients than in HCs $(p<0.05)$. Moreover, the frequencies of CD8+ $T_{E M R A}$ cells and the percentage of divided CD8+ T cells upon CD3 stimulation strongly correlated in GCA baseline patients
$(R=0.79, p=0.009)$ and GCA GC-FR patients $(R=0.67, p=0.039)$ but not in HCs $(\mathrm{R}=0.31, \mathrm{p}=0.25)$

Conclusion: GCA baseline patients demonstrate a higher frequency of proliferating circulating CD8+ $\mathrm{T}_{\mathrm{EM}}$ cells, defined by $\mathrm{Ki}-67$ expression, than $\mathrm{HCs}$. In addition, functional data on induced proliferative capacity suggest that CD8+ $T$ cells from GCA baseline patients are more rapidly activated by crosslinking CD3 and CD3+CD28, suggesting either reduced regulation in these patients or more intrinsic threshold changes. Furthermore, the induced proliferative capacity is also elevated in patients in stable glucocorticoid-free remission. Whether the increased proliferative capacity of total CD8+ T cells in GCA patients is causally linked to the increased frequencies of $C D 8+T_{E M R A}$ cells in these patients requires further investigation.

\section{References:}

[1] Samson M, Ly KH, Tournier B, Janikashvili N, Trad M, Ciudad M, et al. Involvement and prognosis value of CD8+ T cells in giant cell arteritis. J Autoimmun. 2016;72:73-83.

Disclosure of Interests: Rosanne Reitsema: None declared, Rebeca Hid Cadena: None declared, Wayel Abdulahad: None declared, Annemieke Boots Consultant of: Grünenthal Gmbh until 2017, Peter Heeringa: None declared, Elisabeth Brouwer Consultant of: Roche (consultancy fee 2017 and 2018 paid to the UMCG), Speakers bureau: Roche (2017 and 2018 paid to the UMCG)

DOI: 10.1136/annrheumdis-2020-eular.3145

\begin{tabular}{ll}
\hline AB0042 & CYTOKINE NETWORK ELUCIDATED BY THE \\
QUANTIFICATION OF MULTIPLE CYTOKINES IN \\
THE SERUM SEQUENTIALLY SAMPLED FROM RA \\
PATIENTS WHO WERE TREATED WITH BIOLOGIC \\
DMARDS
\end{tabular}

K. Sato ${ }^{1}$, S. Mamada ${ }^{1}$, C. Hayashi ${ }^{1}$, T. Nagashima ${ }^{1}$, S. Minota ${ }^{1} .{ }^{1}$ Jichi Medica University, Division of Rheumatology and Clinical Immunology, Tochigi, Japan

Background: Biologic disease modifying anti-rheumatic drugs (DMARDs) have demonstrated that proinflammatory cytokines such as interleukin (IL-) 6 and tumor necrosis factor (TNF) play important roles in the pathogenesis of rheumatoid arthritis (RA). Other cytokines, such as type I interferons (IFNs), are also implicated in its pathogenesis (ref 1). However, the complete picture of the cytokine network involved in RA remains to be elucidated.

Objectives: By quantifying sets of cytokines in the serum of RA patients before and after treatment with various biologic DMARDs, we sought to determine the effects of drugs on (A) type I IFNs, (B) soluble IL-6 receptors, and (C) other cytokines.

Methods: 52 patients with RA were treated with various biologic DMARDs (tocilizumab (TOC): 16, abatacept (ABT): 15, and TNF inhibitors (TNFi): 21). Serum samples were obtained (1) before, (2) approximately 4 weeks after (3) and approximately 12 weeks after the initiation of treatment. A suspension bead-array system was used for analysis; Bio-Plex Human Cytokine 17-plex Assay kits and Express Custom Panels (Bio-Rad), including IFN- $\beta$, IFN- $\alpha 2$, soluble IL-6 receptor a (sIL6Ra) and gp130 were used.

Results: (1) As expected, the disease activity score 28-joiny count (DAS28) using the erythrocyte sedimentation rate (ESR) significantly decreased in all three groups (TOC, ABT and TNFi) by 12 weeks.

(2) IFN- $\alpha 2$ was barely detected in the serum samples. IFN- $\beta$ seemed to increase slightly in the ABT group, but the increase was not statistically significant.

(3) The levels of sIL6Ra did not change substantially. Those of gp130 decreased slightly but significantly in the TOC group by 12 weeks.

(4) The levels of IL- 6 decreased significantly in the ABT group by 12 weeks. Those in the TNFi group decreased significantly at 4 weeks but not 12 weeks (Fig. 1A) (5) The levels of IL-7 decreased significantly only in the TOC group (Fig. 1B). Conclusion: (1) The biologic DMARDs tested in this study did not significantly affect the serum levels of type I IFNs in this study.

(2) The decrease in gp130 in the TOC group may imply that gp130 is induced by IL-6, although whether this level of decrease has physiological significance is open to question

(3) Serum IL-6 was significantly decreased in the TNFi group at 4 weeks but not 12 weeks. TNF has been reported to induce IL-6 (ref 2), but negative feedback loop(s) may be present. Such a feedback system might make the discontinuation of TNFi difficult, even if patients are in remission.

(4) IL-7 may be a target of IL-6. A higher level of IL-7 has been reported to be present in the joints of RA patients compared with osteoarthrosis and it is a cytokine implicated in the differentiation of osteoclasts (ref 3 ). This may partly explain the effect of TOC on preventing bone erosion in RA.

References:

[1] Ann Rheum Dis. 2007; 66: 1008-14

[2] Rheumatology $2007 ; 46: 920-6$

[3] Rheumatology 2008; 47: 753-9 
Fig. 1A

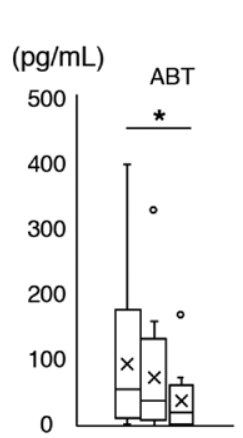

Fig. 1B

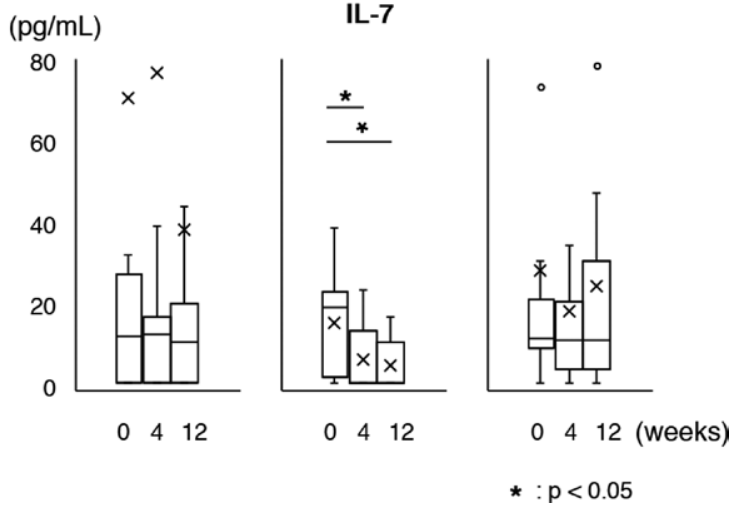

Acknowledgments: We thank all the members of the Division of Rheumatology and Clinical Immunology, Department of Medicine, Jichi Medical University. We are also grateful to the patients involved in this study.

Disclosure of Interests: Kojiro Sato Grant/research support from: Abbie, Pfizer, Chugai, Astellas, Mitsubishi-Tanabe, Ono, Takeda, Sachiko Mamada: None declared, Chiyomi Hayashi: None declared, Takao Nagashima: None declared, Seiji Minota: None declared

DOI: 10.1136/annrheumdis-2020-eular.5109

\section{AB0043 \\ THE IMBALANCE OF T FOLLICULAR REGULATORY CELL ANDT FOLLICULAR HELPER CELL IN RHEUMATOID ARTHRITIS}

R. Su ${ }^{1}$, Y. Y. Wang ${ }^{1}$, F. Y. Hu ${ }^{1}$, X. Zheng ${ }^{1}$, Y. Liu ${ }^{1}$, X. Li ${ }^{1}$, C. Wang ${ }^{1} .{ }^{1}$ Department of Rheumatology, The Second Hospital of Shanxi Medical University, Taiyuan, China

Background: Rheumatoid arthritis (RA) is a chronic inflammatory disease which can lead to severe joint damage and disability. The relationship between antibodies and rheumatoid arthritis has long been well established. Recently, many studies have found that $T$ follicular regulatory cells(Tfr) and T follicular helper cells (Tfh) are closely related to antibody generation on lymphoid follicular germinal centers $(\mathrm{GCs})^{[1-2]}$. Tfr cells can inhibite the GC reaction and suppress production of high-affinity antibodies. The dysregulation of Tfh cells can lead to the production of autoantibodies by B cells.

Objectives: To examine the expression of circulating $T$ follicular regulatory cell (Tfr) and T follicular helper cell and its subsets(Tfh1 Tfh2 Tfh17) in RA patients and healthy control group.

Methods: Level of Tfr and Tfh1,Tfh2 and Tfh17 cells in the peripheral blood of 17 new RA patients, 30 treated RA patients and 18 healthy controls were deceted by flow cytomery. All patients were hospitalised at the Department of Rheumatology, Second Hospital of Shanxi Medical University.

Results: We found that the level of Tfr (CD3+CD4+CD25+CXCR5+FOP3+) percent $(P=0.020)$, in the peripheral blood in $R A$ patients were significantly decreased compared with healthy controls. The percent of Tfh $(C D 3+C D 4+C X-$ CR5+CD45RA- $) \quad(P=0.039)$ and Tfh17 (CD3+CD4+CXCR5+CD45RA-CXCR3-CCR6+) $(P=0.000)$ were increased, but there are no statistical difference about Tfh 1 (CD3+CD4+CXCR5+CD45RA-CXCR3+CCR6-) $(P=0.558)$ and Tfh2 (CD3+CD4+CXCR5+CD45RA-CXCR3-CCR6-) percent $(P=0.079)$. We compared the above indicators between new and treated RA patients, and the results indicated that the $\operatorname{Tr}(P=0.013)$, $\operatorname{Tfh}(P=0.002)$ and $\operatorname{Tfh} 1(P=0.034)$ were significantly increased in the new RA patients compared to the treated RA patients, there were no differences between the two groups in Tfh2 $(P=0.419)$ and Tfh17 percent $(P=0.124)$.

Conclusion: Our results indicated that disorder of Tfr and Th subsets were involved in RA, restoring the Tfr/Tfh balance may be the potential therapeutic targets.
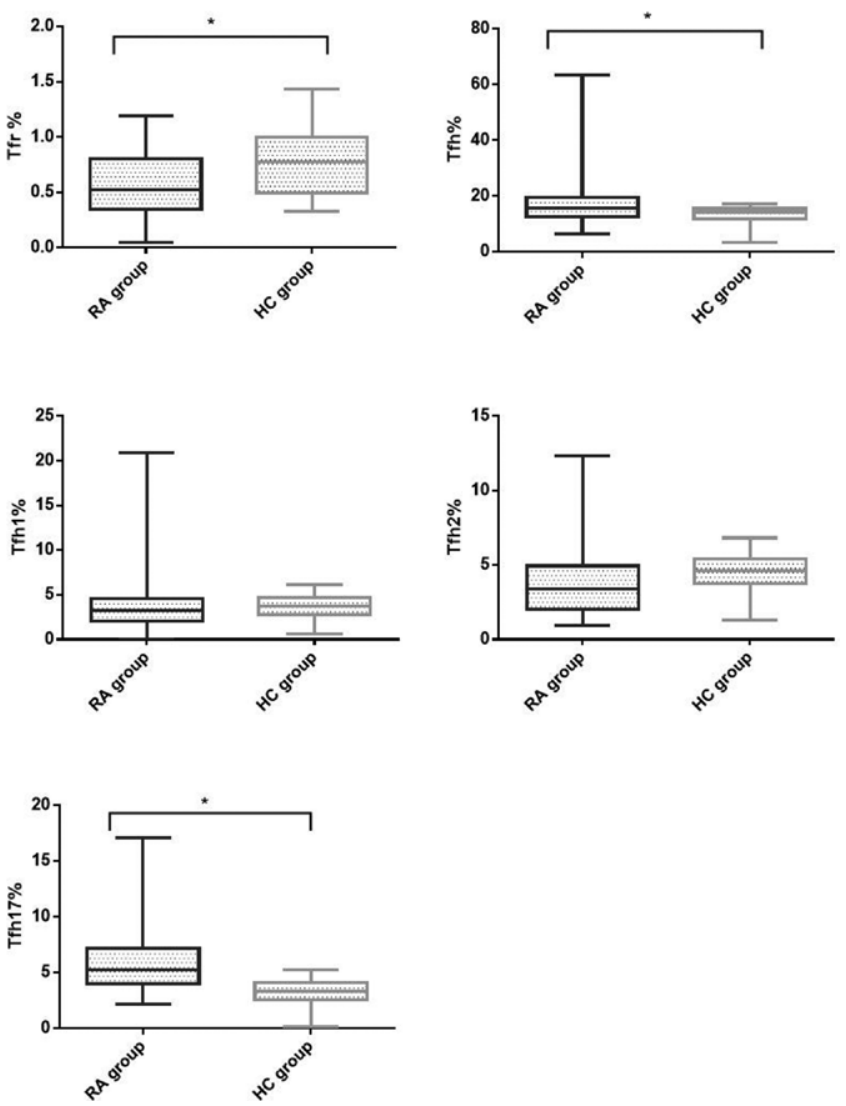

Fig. 1. Comparison of Tfr, Tfh and its subsets(Tfh1 Tfh2 Tfh17) percent among the RA patients $(n=47)$ and healthy control group $(n=18)\left({ }^{*} P<0.05\right)$.
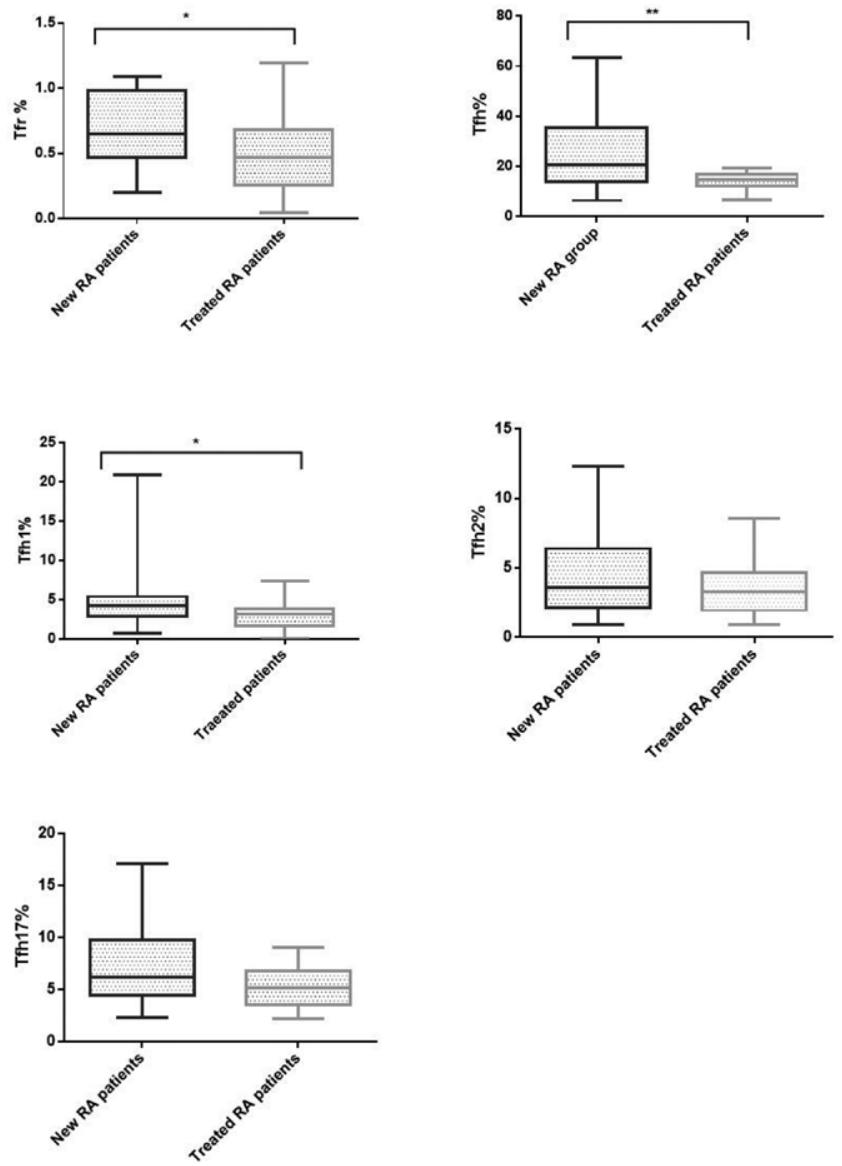

Fig. 2. Comparison of Tfr,Tfh and its subsets(Tfh1 Tfh2 Tfh17) percent among the new RA patients $(n=17)$ and treated RA patients $(n=30)\left({ }^{*} P<0.05\right)$ 